diagnosed. The first round detected 13 regional and 9 distant metastases, but only 2 distant metastases were detected in the second round. In the second round, mean PSA values were lower $(5.6 \mathrm{ng} / \mathrm{ml}$ versus $11.1 \mathrm{ng} / \mathrm{ml})$, advanced (T3-T4) clinical stage cancers were 7.1-fold less common, and $76.4 \%$ versus $61.5 \%$ of biopsy Gleason scores were $<7$ than in the first round.

These results demonstrate a shift towards more favorable tumor characteristics and prognostic factors in the second round of screening. At present, the optimal screening interval is unclear, although this study supports the interval of 4 years.

Original article van der Cruijsen-Koeter IW et al. (2006) Tumor characteristics and prognostic factors in two subsequent screening rounds with four-year interval within prostate cancer screening trial, ERSPC Rotterdam. Urology 68: 615-620

\section{A new treatment for girls with primary nocturnal enuresis}

Several approaches have been used to treat the multifactorial condition, primary nocturnal enuresis (PNE), without complete success. Functional magnetic stimulation (FMS) has been suggested as an alternative to electrical stimulation for the treatment of bladder overactivity in women. But and Marcun Varda carried out a pilot study to establish the efficacy of FMS in girls with PNE.

This randomized, placebo-controlled, doubleblind study included 20 girls with PNE (age range $6-14$ years). The girls were randomly assigned to active or placebo Pulsegen devices and instructed to wear the device continuously for 2 months, while keeping a voiding diary. The active Pulsegen device generated a low-frequency pulsating electromagnetic field.

A significant improvement in PNE episodes was seen after FMS treatment $(P=0.007)$. The number of PNE episodes in the active-treatment group fell from 3.1 to 1.3 per week. After treatment, three girls from the active-treatment group experienced no PNE episodes, compared with one girl from the placebo-treatment group. In the active-treatment group, a significant increase in bladder volume at maximal desire to void was observed $(P=0.022)$ and there was also a trend towards increased bladder volume at first desire to void. Patients did not report any discomfort or adverse effects.

This study confirms that FMS is safe and suitable for use in children. Further randomized, placebo-controlled studies in larger numbers of girls are recommended to confirm these results. Studies to evaluate the long-term efficacy of this promising treatment are also needed.

Original article But I and Marcun Varda N (2006) Functional magnetic stimulation: a new method for the treatment of girls with primary nocturnal enuresis? J Pediatr Urol 2: 415-418

\section{Testosterone gel and progestin show promise as a male hormonal contraceptive}

Administration of exogenous androgens and progestins suppress pituitary secretion of sex hormones and inhibit spermatogenesis, but only injectable or implantable testosterone had previously shown efficacy. Page and colleagues have now demonstrated that transdermal testosterone gel plus depomedroxyprogesterone acetate (DMPA, a progestin) induced severe oligospermia in $80-90 \%$ of treated men; they also confirmed that acyline (a gonadotropin-releasing-hormone antagonist that directly suppresses pituitary sex hormones) did not improve the efficacy or rapidity of spermatogenesis suppression achieved by testosterone gel plus DMPA.

Their open-label study involved 44 men aged 18-55 years with normal spermatogenesis, who were randomly allocated to 24 weeks of treatment $(100 \mathrm{mg}$ of $1 \%$ testosterone gel self-applied daily, plus $300 \mathrm{mg}$ intramuscular DMPA once every 3 months, with or without 12 weeks of $300 \mu \mathrm{g} / \mathrm{kg}$ subcutaneous acyline once every 2 weeks). Patients were evaluated at monthly intervals. The protocol was completed by $17 / 22$ acyline-treated and $21 / 22$ nonacyline-treated men. Sperm count declined to $\leq 1 \mathrm{million} / \mathrm{ml}$ after a mean of $7-9$ weeks in both groups, and had normalized by a mean of 11-15 weeks after treatment ceased. There were no serious adverse events, and changes in body weight, serum lipids and PSA were minimal and reversible.

The authors conclude that transdermal testosterone gel plus DMPA is well tolerated and effective; they call for extended-duration studies to determine the dose of testosterone gel required to optimize spermatogenesis suppression.

\footnotetext{
Original article Page ST et al. (2006) Testosterone gel combined with depomedroxyprogesterone acetate is an effective male hormonal contraceptive regimen and is not enhanced by the addition of a GnRH antagonist. J Clin Endocrinol Metab [doi:10.1210/jc.2006-1411]
} 